\title{
Invariant representation for spectral reflectance images and its application
}

\author{
Abdelhameed Ibrahim ${ }^{1,2^{*}}$, Shoji Tominaga ${ }^{1}$ and Takahiko Horiuchi ${ }^{1}$
}

\begin{abstract}
Spectral images as well as color images observed from object surfaces are much influenced by various illumination conditions such as shading and specular highlight. Several invariant representations were proposed for these conditions using the standard dichromatic reflection model of dielectric materials. However, these representations are inadequate for other materials like metal. This article proposes an invariant representation that is derived from the standard dichromatic reflection model for dielectric and the extended dichromatic reflection model for metal. We show that a normalized surface-spectral reflectance by the minimum reflectance is invariant to highlights, shading, surface geometry, and illumination intensity. Here the illumination spectrum and the spectral sensitivity functions of the imaging system are measured in a separate way. As an application of the proposed invariant representation, a segmentation algorithm based on the proposed representation is presented for effectively segmenting spectral images of natural scenes and bare circuit boards.
\end{abstract}

Keywords: Invariant representation, dichromatic reflection models, spectral imaging system, spectral image segmentation

\section{Introduction}

Spectral images contain large amount of information, compared with color images, are useful for a variety of applications such as material identification, natural scene rendering, colorimetric analysis, and machine vision tasks [1]. Spectral images as well as color images observed from object surfaces are much influenced by various illumination conditions. The observed spectral images do not only depend on surface-spectral reflectance and illuminant spectrum, but also include various reflection effects such as shading and specularity, which mainly depend on illumination geometries and surface materials. Therefore, image representations invariant to shading, shadow, lighting, and specularity have been proposed for color images [2-6] and for spectral images $[7,8]$ so far in several ways.

Geusebroek et al. [3] investigated the differential photometric invariance. Van de Weijer et al. [4] introduced the photometric quasi-invariants which were a set of photometric invariant derivatives with better noise

\footnotetext{
* Correspondence: afai79@yahoo.com

'Graduate School of Advanced Integration Science, Chiba University, 1-33,

Yayoi-cho, Inage-ku, Chiba, 263-8522, Japan

Full list of author information is available at the end of the article
}

and stability characteristics. Combining the photometric quasi-invariants with derivative-based feature detectors led to features which could identify shadow corners and object corners [5]. Stokman and Gevers [7] proposed a method for edge classification from spectral images. Their method aimed at detecting edges and assigning one of the types of shadow, highlight, and material edge. However, most of those methods were constructed based on the standard dichromatic reflection model by Shafer [9]. This model assumes that an object surface is composed of inhomogeneous dielectric material, and reflected light from the surface is decomposed into two additive components of body (diffuse) reflection and interface (specular) reflection. This decomposition results in the classification of physics events, such as shadows and highlights. However, the model-based method is valid for such limited materials as plastics and paints [10-12]. It should be noted that there are metallic objects in real-world scenes, which cannot be described by the standard dichromatic reflection model.

The present article proposes an invariant representation for spectral reflectance images. The invariant representation for a variety of objects in a real world is derived from the standard dichromatic reflection model

\section{SpringerOpen ${ }^{\circ}$}


for dielectric [9] and the extended dichromatic reflection model for metal $[13,14]$. We show that the invariant formulas for spectral images of both artificial object and natural ones preserve surface-spectral reflectance information and are invariant to highlight, shadow, surface geometry, and illumination intensity. Here we measured the illumination spectrum by using a Spectro-radiometer and a standard white reference and measured the spectral sensitivity functions of the imaging system by using a Spectro-radiometer and a Monochromator.

As an application of the proposed invariant representation, the transformed spectral image with the invariant properties is used as an invariant operator for the image segmentation problem. Several algorithms were recently proposed for segmenting the spectral images into distinct surface areas [15-19]. However, those algorithms were not always robust for highlight and shading effects occurred for different surface materials, and required a careful adjustment of the lighting position. As a typical example, it was difficult to segment materials on a bare circuit board with various tiny elements [20-23]. In this article, we present a segmentation algorithm based on the proposed representation for effectively segmenting spectral images of natural scenes and bare circuit boards. The proposed invariant representation is also applied to other alternative segmentation algorithms. Experimental results using real-world objects including metals and dielectrics show that the proposed representation is invariant to highlight, shadow, and object surface geometry, and effective for image segmentation.

The reminder of this article is organized as follow: 'Dichromatic reflection models' section describes two types of dichromatic reflection models. 'Proposed invariant representation' section develops the proposed invariant representation in detail. 'Test of invariant representation' section tests the invariant properties for the captured spectral images of several sample objects. 'Application to image segmentation' section discusses image segmentation based on the transformed spectral images to the invariant representation. This article ends with 'Conclusions' section.

\section{Dichromatic reflection models}

The standard dichromatic reflection model [9] suggests that light reflected from the surface of an inhomogeneous dielectric object is composed of two additive components, the interface reflection and the body reflection. The radiance of the reflected light $Y(\theta, \lambda)$ is a function of the wavelength $\lambda$, ranging over a visible wavelength 400 to $700 \mathrm{~nm}$, and the geometric parameters $\theta$, including the direction angles of the viewing angle and the phase angle. The standard dichromatic reflection model describes the reflected light in the form

$$
Y(\theta, \lambda)=c_{\mathrm{I}}(\theta) L_{\mathrm{I}}(\lambda)+c_{\mathrm{B}}(\theta) L_{\mathrm{B}}(\lambda)
$$

where $L_{\mathrm{I}}(\lambda)$ and $L_{\mathrm{B}}(\lambda)$ are the spectral power distributions of the interface and the body reflection components, respectively. The weights $\mathrm{c}_{\mathrm{I}}(\theta)$ and $\mathrm{c}_{\mathrm{B}}(\theta)$ are geometric scale factors.

The reflection model is also described in terms of spectral reflectance. Let $E(\lambda)$ be the spectral-power distribution of the incident light. The spectral reflectance function defined as $S(\theta, \lambda)=Y(\theta, \lambda) / E(\lambda)$, independent of illumination, can be expressed as

$$
S(\theta, \lambda)=c_{\mathrm{I}}(\theta) S_{\mathrm{I}}(\lambda)+c_{\mathrm{B}}(\theta) S_{\mathrm{B}}(\lambda)
$$

where $S_{\mathrm{I}}(\lambda)$ and $S_{\mathrm{B}}(\lambda)$ are surface-spectral reflectances for the interface and the body components, respectively. The standard model incorporates the neutral interface reflection (NIR) assumption which states that the interface reflection component $S_{\mathrm{I}}(\lambda)$ is constant over the range of visible wavelength as $S_{\mathrm{I}}(\lambda)=S_{\mathrm{I}}^{\mathrm{c}}$. This allows Equation 2 to be written as

$$
S(\theta, \lambda)=c_{\mathrm{I}}^{\prime}(\theta)+c_{\mathrm{B}}(\theta) S_{\mathrm{B}}(\lambda)
$$

where $c_{\mathrm{I}}^{\prime}(\theta)=c_{\mathrm{I}}(\theta) S_{\mathrm{I}}^{\mathrm{c}}$. It is shown that this reflectance model is valid for a variety of natural and artificial dielectric objects including plastic and paint (e.g., see [10-12]).

Metal is a homogeneous material that indicates essentially different reflection properties from the inhomogeneous dielectric materials. It consists of only interface reflection with the Fresnel reflectance. Therefore, if the surface is shiny and stainless, the body reflection component in the reflected light is negligibly small. A sharp specular highlight is observed only at the viewing angle of the mirrored direction. Thus, the surface reflection depends on the incident angle of illumination. Tominaga [13] shows that the surface-spectral reflectance function of a metal can be approximated by a linear combination of two interface reflection components as

$$
S(\theta, \lambda)=c_{I 1}(\theta) S_{I}(\lambda)+c_{I 2}^{\prime}(\theta)
$$

where the first term on the right hand side corresponds to the specular reflectance at the normal incident. The second term, which is constant over the visible wavelength range, corresponds to the grazing reflection at the horizontal incident. It is important to note that the observed spectral reflectance can be expressed in a linear combination of the reflectance function at the normal incidence and a constant reflectance. This type of surface reflection is called the extended dichromatic reflection model.

\section{Proposed invariant representation}

We briefly explain an invariant representation for spectral images $[7,8]$, which was limited to the standard 
reflection model for inhomogeneous dielectric objects, and then extend the invariant representation for all materials including inhomogeneous dielectric and homogeneous metal.

Let us suppose that a spectral image is captured at $N$ points in the visible range 400 to $700 \mathrm{~nm}$. Let $i$ and $j$ be two different wavelengths (spectral bands) in the range. From Equation 3 of the standard reflection model, subtraction of one band from another provides a reflectance representation that is independent of specular highlight as

$$
\begin{aligned}
S\left(\theta, \lambda_{i}\right)-S\left(\theta, \lambda_{j}\right) & =\left(c^{\prime}(\theta)+c_{\mathrm{B}}(\theta) S_{\mathrm{B}}\left(\lambda_{i}\right)\right)-\left(c_{\mathrm{I}}^{\prime}(\theta)+c_{\mathrm{B}}(\theta) S_{\mathrm{B}}\left(\lambda_{j}\right)\right) \\
& =c_{\mathrm{B}}(\theta)\left(S_{\mathrm{B}}\left(\lambda_{i}\right)-S_{\mathrm{B}}\left(\lambda_{j}\right)\right)
\end{aligned}
$$

Moreover let $l$ and $n$ be two other wavelengths. Then the following ratio of two subtractions between wavelengths can be spectral invariant, that is, invariant to highlight, shading, and surface geometry $[7,8]$.

$$
\begin{aligned}
\frac{S\left(\theta, \lambda_{i}\right)-S\left(\theta, \lambda_{j}\right)}{S\left(\theta, \lambda_{l}\right)-S\left(\theta, \lambda_{n}\right)} & =\frac{c_{\mathrm{B}}(\theta)\left(S_{\mathrm{B}}\left(\lambda_{i}\right)-S_{\mathrm{B}}\left(\lambda_{j}\right)\right)}{c_{\mathrm{B}}(\theta)\left(S_{\mathrm{B}}\left(\lambda_{l}\right)-S_{\mathrm{B}}\left(\lambda_{n}\right)\right)} \\
& =\frac{S_{\mathrm{B}}\left(\lambda_{i}\right)-S_{\mathrm{B}}\left(\lambda_{j}\right)}{S_{\mathrm{B}}\left(\lambda_{l}\right)-S_{\mathrm{B}}\left(\lambda_{n}\right)}
\end{aligned}
$$

Next, we newly consider the reflection model of metal objects. The extended reflection model in Equation 4 is the same fashion mathematically as the standard model in Equation 3 for dielectric, although the two reflection models are physically different. Therefore, we derive a unified invariant representation for all materials including inhomogeneous dielectric and homogeneous metal. In fact, the following invariant equation can be derived for metal from Equation 4,

$$
\begin{aligned}
\frac{S\left(\theta, \lambda_{i}\right)-S\left(\theta, \lambda_{j}\right)}{S\left(\theta, \lambda_{l}\right)-S\left(\theta, \lambda_{n}\right)} & =\frac{C_{\mathrm{I} 1}(\theta)\left(S_{\mathrm{I}}\left(\lambda_{i}\right)-S_{\mathrm{I}}\left(\lambda_{j}\right)\right)}{C_{\mathrm{I} 1}(\theta)\left(S_{\mathrm{I}}\left(\lambda_{l}\right)-S_{\mathrm{I}}\left(\lambda_{n}\right)\right)} \\
& =\frac{S_{\mathrm{I}}\left(\lambda_{i}\right)-S_{\mathrm{I}}\left(\lambda_{j}\right)}{S_{\mathrm{I}}\left(\lambda_{l}\right)-S_{\mathrm{I}}\left(\lambda_{n}\right)}
\end{aligned}
$$

where the geometric weighting coefficients $c_{\mathrm{I} 1}(\theta)$ and $c^{\prime}{ }_{\text {I2 }}(\theta)$ in Equation 4 are eliminated. We note that the operation of Equation 7 results in an equivalent operation to Equation 6, that depends on only surface-spectral reflectance and is invariant to highlights, shading, and geometries.

The above spectral operations based on subtraction and division provides mathematically simple and robust spectral invariant representations. However, the above computation depends on wavelengths such as $\lambda_{l}$ and $\lambda_{n}$, and therefore, for real spectral data it is unstable because the denominator includes subtraction and we can get negative values. There is no method that enables us to choose the appropriate bands. Thus, the invariant is unstable and depends on wavelengths. The above operation can be generalized to a stable form. Note that use of the minimum value of spectral reflectance preserves the original spectral characteristics. Then an invariant representation for spectral reflectance is defined as

$$
S^{\prime}(\theta, \lambda)=\frac{S(\theta, \lambda)-\min \{S(\theta, \lambda)\}}{\sqrt{\int_{400}^{700}(S(\theta, \lambda)-\min \{S(\theta, \lambda)\})^{2} \mathrm{~d} \lambda}}
$$

For practical computation, let us sample spectral reflectance at $N$ wavelengths in the visible range 400 to $700 \mathrm{~nm}$. Then the discrete version of the invariant representation is written as

$$
S^{\prime}\left(\theta, \lambda_{i}\right)=\frac{S\left(\theta, \lambda_{i}\right)-\min \left\{S\left(\theta, \lambda_{1}\right), \ldots, S\left(\theta, \lambda_{N}\right)\right\}}{\sqrt{\sum_{j=1}^{N}\left(S\left(\theta, \lambda_{j}\right)-\min \left\{S\left(\theta, \lambda_{1}\right), \ldots, S\left(\theta, \lambda_{N}\right)\right\}\right)^{2}}} \quad(i=1,2, \ldots, N)
$$

This representation is spectral invariant for all materials, including inhomogeneous dielectric and homogeneous metal. The observed spectral reflectance $S(\theta, \lambda)$ can be reduced into a normalized surface-spectral reflectance $S^{\prime}(\theta, \lambda)=S^{\prime}(\lambda)$ that is independent of the geometric parameter $\theta$. This representation is useful as an invariant operator for a variety of spectral image analysis, including material classification and image segmentation. The properties are summarized in the following lemmas.

Lemma 1: Assuming the standard dichromatic reflection model for inhomogeneous dielectric materials, $S^{\prime}(\theta$, $\lambda)$ is independent of highlight, shading, surface geometry, and illumination intensity.

Proof: By substituting Equation 3 in Equation 9 and factoring out dependencies on highlight, shading, surface geometry, and illumination intensity, we have

$$
\begin{aligned}
S^{\prime}\left(\theta, \lambda_{i}\right)= & \frac{c_{\mathrm{B}}(\theta) S_{\mathrm{B}}\left(\lambda_{i}\right)-\min \left\{c_{\mathrm{B}}(\theta) S_{\mathrm{B}}\left(\lambda_{1}\right), \ldots, c_{\mathrm{B}}(\theta) S_{\mathrm{B}}\left(\lambda_{N}\right)\right\}}{\sqrt{\sum_{j=1}^{N}\left(c_{\mathrm{B}}(\theta) S_{\mathrm{B}}\left(\lambda_{j}\right)-\min \left\{c_{\mathrm{B}}(\theta) S_{\mathrm{B}}\left(\lambda_{1}\right), \ldots, c_{\mathrm{B}}(\theta) S_{\mathrm{B}}\left(\lambda_{N}\right)\right\}\right)^{2}}} \\
& =\frac{c_{\mathrm{B}}(\theta)\left(S_{\mathrm{B}}\left(\lambda_{i}\right)-\min \left\{S_{\mathrm{B}}\left(\lambda_{1}\right), \ldots, S_{\mathrm{B}}\left(\lambda_{N}\right)\right\}\right)}{c_{\mathrm{B}}(\theta) \sqrt{\sum_{j=1}^{N}\left(S_{\mathrm{B}}\left(\lambda_{j}\right)-\min \left\{S_{\mathrm{B}}\left(\lambda_{1}\right), \ldots, S_{\mathrm{B}}\left(\lambda_{N}\right)\right\}\right)^{2}}} \\
& =\frac{S_{\mathrm{B}}\left(\lambda_{i}\right)-\min \left\{S_{\mathrm{B}}(\lambda)\right\}}{\sqrt{\sum_{j=1}^{N}\left(S_{\mathrm{B}}\left(\lambda_{j}\right)-\min \left\{S_{\mathrm{B}}(\lambda)\right\}\right)^{2}}}(i=1,2, \ldots, N)
\end{aligned}
$$

Lemma 2: Assuming the extended dichromatic reflection model for homogeneous metal, $S^{\prime}(\theta, \lambda)$ is independent of highlight, shading, surface geometry, and illumination intensity.

Proof: By substituting Equation 4 in Equation 9 and factoring out dependencies, we have

$$
\begin{aligned}
S^{\prime}\left(\theta, \lambda_{i}\right) & =\frac{c_{11}(\theta) S_{\mathrm{I}}\left(\lambda_{i}\right)-\min \left\{c_{11}(\theta) S_{\mathrm{I}}\left(\lambda_{1}\right), \ldots, c_{11}(\theta) S_{\mathrm{I}}\left(\lambda_{N}\right)\right\}}{\sqrt{\sum_{j=1}^{N}\left(c_{11}(\theta) S_{\mathrm{I}}\left(\lambda_{j}\right)-\min \left\{c_{11}(\theta) S_{\mathrm{I}}\left(\lambda_{1}\right), \ldots, c_{11}(\theta) S_{\mathrm{I}}\left(\lambda_{N}\right)\right\}\right)^{2}}} \\
& =\frac{c_{11}(\theta)\left(S_{\mathrm{I}}\left(\lambda_{i}\right)-\min \left\{S_{\mathrm{I}}\left(\lambda_{1}\right), \ldots, S_{\mathrm{I}}\left(\lambda_{N}\right)\right\}\right)}{c_{11}(\theta) \sqrt{\sum_{j=1}^{N}\left(S_{\mathrm{I}}\left(\lambda_{j}\right)-\min \left\{S_{\mathrm{I}}\left(\lambda_{1}\right), \ldots, S_{\mathrm{I}}\left(\lambda_{N}\right)\right\}\right)^{2}}} \\
& =\frac{S_{\mathrm{I}}\left(\lambda_{i}\right)-\min \left\{S_{\mathrm{I}}(\lambda)\right\}}{\sqrt{\sum_{j=1}^{N}\left(S_{\mathrm{I}}\left(\lambda_{j}\right)-\min \left\{S_{\mathrm{I}}(\lambda)\right\}\right)^{2}}},(i=1,2, \ldots, N)
\end{aligned}
$$




\section{Test of invariant representation}

\section{Spectral imaging system}

The proposed invariant representation was tested for spectral images of different metal and dielectric objects. Use of a liquid crystal tunable (LCT) filter provides precise filtration for spectral imaging [24-27]. We have developed a spectral imaging system, shown in Figure 1a, which consists of a monochromatic CCD camera with an air cooling system and 12-bit dynamic range, a VariSpect LCT filter, an IR-cut filter, and a personal computer. Accuracy decreases when spectral sensors have certainly broadband. Our system with LCT filter has the spectral properties of narrowband filtration of $10 \mathrm{~nm}$ and wavelength range 400 to $700 \mathrm{~nm}$. This is not ideally narrowband, but excellent result can be obtained. The LCT filter tune to any wavelength in the visible range without vibration or noise.

The imaging system automatically captures and saves spectral images with arbitrary number of bands and shutter speeds. The actual measurement time required for capturing one spectral image with 31 bands is $4.75 \mathrm{~s}$. Incandescent lamps are used for effective surface illumination. Figure 1b shows the total spectral sensitivity functions of the imaging system. We measured the spectral sensitivity functions of the imaging system by using a Spectro-radiometer and a Monochromator. Spectral images calibration to compensate the undesirable effects due to uncontrolled acquisition conditions is done using a standard white reference and a black reference. The camera noise is captured using the black reference.

The dynamic range of 12-bits looks adequate for imaging of most objects surfaces. However, when we observe object surfaces including strong specular highlights, such as smooth plastic and metal surfaces, we need high-dynamic range (HDR) imaging. The HDR imaging allows spectral images to more accurately represent the range of intensity levels found in real scenes. Without HDR, areas that are too dark are clipped to black and areas that are too bright are clipped to white. This will strongly affect the invariant representation results. In this case, we extend the original dynamic range as follow: Suppose that three pictures of the same scene are automatically taken with different shutter speeds (different exposures). Then we combine the multiple images into a single image to extend the dynamic range of the capture image to 14-bits equivalently.

If the illumination spectrum is known in advance, the surface-spectral reflectance can be estimated in a straightforward way from the camera outputs under narrowband filtration. Let $\lambda_{1}, \lambda_{2, \ldots}, \lambda_{31}$ be 31 -wavelengths of filtration. The surface-spectral reflectance can be estimated from the sensor outputs $Y\left(\theta, \lambda_{1}\right), Y\left(\theta, \lambda_{2}\right), \ldots, Y(\theta$, $\left.\lambda_{31}\right)$, as

$$
S\left(\theta, \lambda_{i}\right)=\frac{Y\left(\theta, \lambda_{i}\right)}{\int_{400}^{700} E(\lambda) R_{i}(\lambda) \mathrm{d} \lambda}, \quad(i=1,2, \ldots, 31)
$$

where $E(\lambda)$ is the illuminant spectral-power distribution, and $R_{i}(\lambda)$ is the $i$-th sensor spectral sensitivity function in Figure 1b. The sensor output at each wavelength in Equation 12 is normalized with a factor on illuminant and sensing sensitivity. Thus, the spectral reflectance is recovered by eliminating the lighting and sensing effects from the sensor outputs. In a general computer vision problem we have to estimate the illumination spectrum. This problem is discussed in 'Illumination estimation and invariant representation' section.

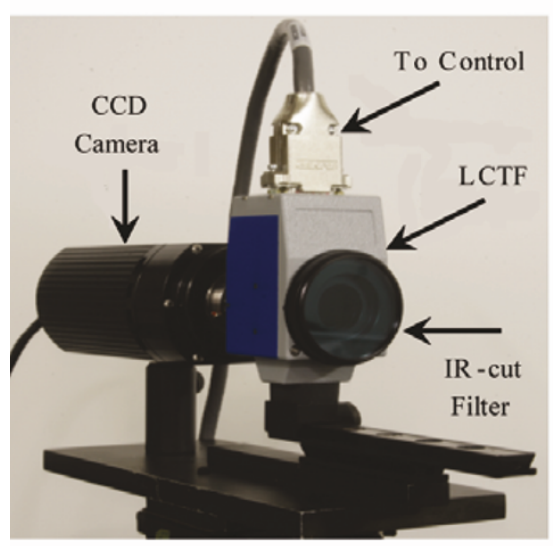

(a)

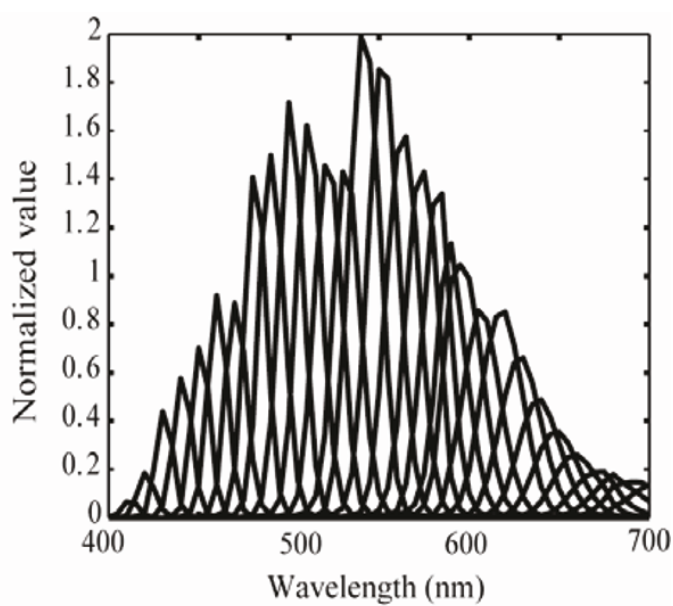

(b)

Figure 1 Proposed spectral imaging system. (a) system overview; (b) total spectral-sensitivity functions. 


\section{Test result}

We have used a test scene including a metal object of copper and two dielectric objects of ceramic (cup) and plastic (frog). Figure 2a shows the color image of the original surface-spectral reflectances. This color image was obtained using the CIE-color matching functions, which are the spectral sensitivity curves of the CIE XYZ tristimulus values $\mathrm{X}, \mathrm{Y}$, and $\mathrm{Z}$, of the observed reflectance $S(\theta, \lambda)$. Then the XYZ-space was converted to a RGB-space. We note the scene has the illumination effects of shadows and highlights observed over different parts on the object surfaces. From this scene, we obtained a set of spectral reflectance images with the size of $439 \times 297 \times 31$. Figure $2 b$ shows the color image of the proposed invariant representation of the normalized spectral reflectance. Shadows, highlights, and surface geometry are much reduced in the invariant representation of both the dielectric objects and the metal object.

For detailed inspection, Figure 2c depicts a $3 \mathrm{D}$ view of the component image of spectral reflectance at $550 \mathrm{~nm}$ for a small rectangular area including metal and dielectrics. The observed reflectance $S(\theta, \lambda)$ is strongly influenced by shading and illumination effects. Figure $2 \mathrm{~d}$

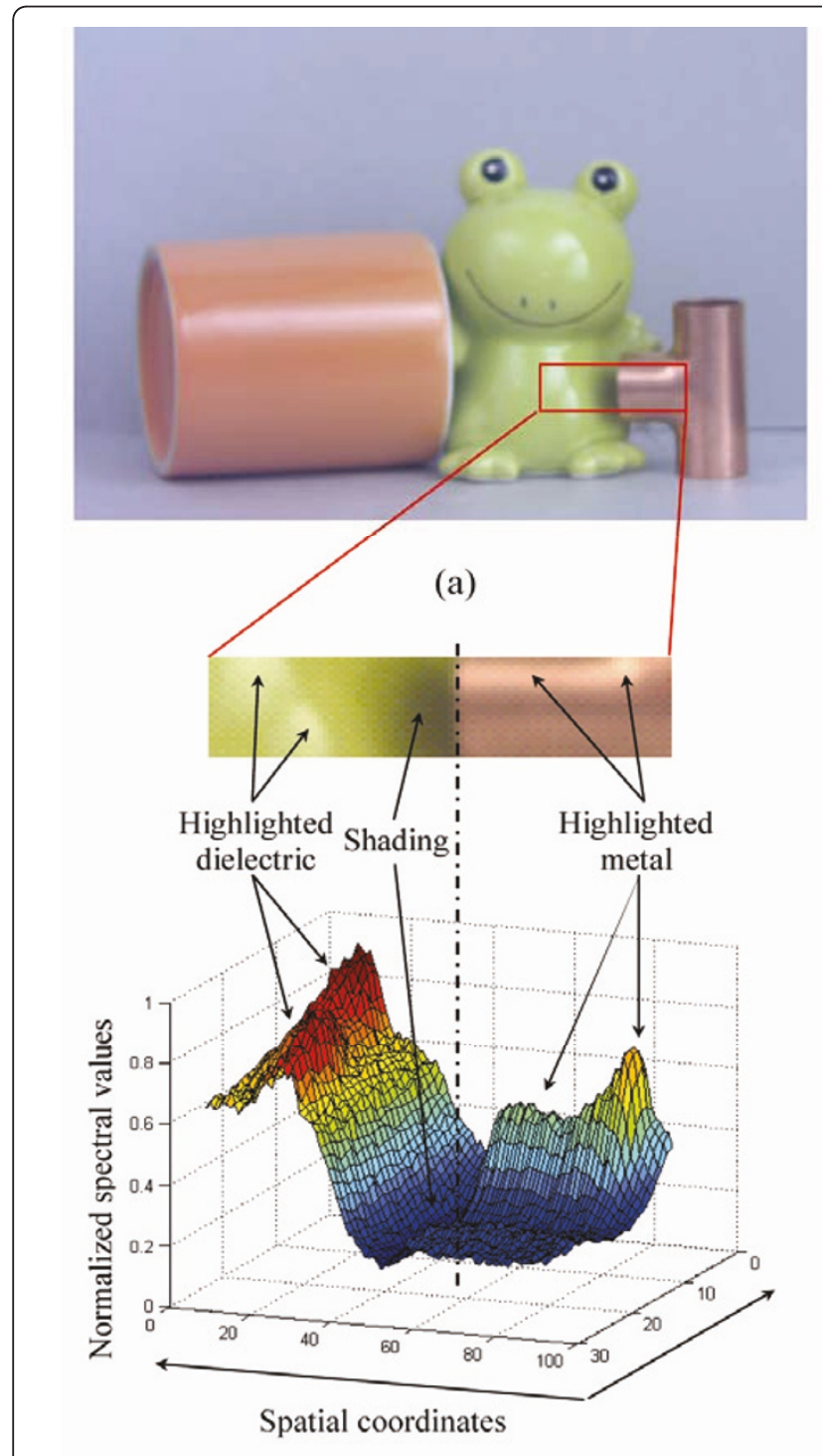

(c)



(d)

Figure 2 Invariant evaluation for spectral reflectance image including dielectric and metal objects. (a) color image of the original spectral reflectances; (b) color image of the proposed invariant representation; (c) 3D view of the component image of spectral reflectance at $550 \mathrm{~nm}$ for a small area including metal and dielectrics; (d) 3D view of the invariant representation for the same part at $550 \mathrm{~nm}$. 
shows the 3D view of the invariant representation $S^{\prime}(\theta$, $\lambda)=S^{\prime}(\lambda)$ for the same part at $550 \mathrm{~nm}$. Shadows and highlights disappear from the test part, so that the transformed spectral image depends on only the inherent spectral reflectance to each object surface. Therefore, the image is clearly segmented into two different material regions. Thus, the proposed invariant representation is valid for both dielectric and metal objects, and is much more robust under a variety of illumination effects than the observed reflectance data.

To confirm the effectiveness of the proposed invariant representation, we tested another spectral image including a metal object and two dielectric objects of plastic. Figure 3a shows the color image of the original surfacespectral reflectances. The image has the illumination effects of shadows and highlights. Figure $3 \mathrm{~b}$ shows the color image of the proposed invariant representation. We note that shadows, highlights, and surface geometry are much reduced in the invariant representation of both the dielectric objects and the metal object.

\section{Application to image segmentation Algorithms for spectral image segmentation}

As an application of the proposed invariant representation, the transformed spectral image with the invariant properties is used as an invariant operator for the image segmentation problem. Several algorithms have been proposed for segmenting the spectral images into distinct surface areas [15-19]. Paclík et al. [15] proposed a segmentation method of spectral images acquired by imaging cross-sections with scanning electron microscopy using energy-dispersive X-ray microanalysis (SEM/EDX). An effective method was introduced by Martínez-Usó et al. [16] for spectral image segmentation in fruit inspection applications. A hierarchical Markovian model for hyperspectral image segmentation was proposed by Mohammad-Djafari et al. [17]. However, those algorithms were not always robust for the highlight and shading effects occurred for different surface materials, and required a careful adjustment of the lighting position.

In our previous study, we introduced a segmentation algorithm 'PCA +Ncut method' for material classification using dimension-reduced spectral information [18]. This method was used to execute the normalized cut (Ncut) [28] in a low-dimensional space obtained with the principal components analysis (PCA). In Ref. [19], we proposed another algorithm which was fundamentally based on the normalized cut approximated by the Nyström method [29] to incorporate both spectral information and spatial information. Those previous methods were useful for material classification of printed circuit boards. However, the material classification was not invariant to illumination effects, but was performed under the ideal lighting condition of eliminating specular reflection and shadow.

In this article, we introduce an image segmentation algorithm based on the proposed invariant representation, which results in reliable segmentation results for natural scenes and circuit boards under arbitrary lighting conditions. The image segmentation process is composed of the following four steps:

(1) The surface-spectral reflectances are estimated from the camera outputs, and then transformed into the invariant representation to highlight, shadow, and surface geometry.

(2) The similarity matrix between pixels is constructed in the normalized cut scheme.

(3) The computation burden is reduced by the Nyström approximation.

(4) The $K$-means algorithm is applied to cluster the leading normalized eigenvectors to get the final segmentation.

\section{Illumination estimation and invariant representation}

The estimation of scene illumination from image data is one of interesting topics in image analysis and computer vision [1]. Although many algorithms were proposed for



(a)



(b)

Figure 3 Invariant evaluation for spectral reflectance image including dielectric and metal objects. (a) color image of the original spectral reflectances; (b) color image of the proposed invariant representation. 
scene illuminant estimation, most algorithms assumed spatially uniform illumination from a single light source $[30,31]$. Concerning spectral imaging, we presented an illuminant estimation method from the outputs of a multi-channel vision system [26,30], where the dichromatic reflection model was assumed to object surfaces. Therefore the illuminant spectrum could be estimated from the interface reflection component. Recently, the authors proposed a method for estimating the illuminant spectral-power distributions from omnidirectional observations by a multichannel omnidirectional imaging system $[32,33]$.

An easy way to estimate the illuminant spectrum is to place a standard white reference in a scene and capture the corresponding sensor outputs from the spectral image of the scene. The sensor outputs are then described as $\int_{400}^{700} E(\lambda) R_{i}(\lambda) \mathrm{d} \lambda(i=1,2, \ldots, 31)$. When the spectral sensitivities $R_{i}(\lambda)$ are narrow, the illuminant is estimated directly from the sensor outputs. This method is utilized in inspection systems for industrial application.

Here suppose that the spatially uniform illuminant spectrum $E(\lambda)$ is estimated from multiple light sources by such a method as described in the above. Then the surface-spectral reflectance $S(\theta, \lambda)$ of an object is obtained in a straightforward way using Equation 12. Moreover, the normalized spectral reflectance $S^{\prime}(\lambda)$ for invariant representation is obtained from the transformation of Equation 9. The following segmentation method is adapted to the invariant representation for classifying each pixel to a specific object.

\section{Similarity matrix construction in the normalized cut scheme} In the normalized cut scheme, a similarity matrix $\mathbf{W}$ is constructed by all combination of two pixels based on the input $\mathrm{N}$-dimensional feature vector, which corresponds to the invariant representation. The similarity $w$ $(x, y) \in \mathbf{W}$ between two pixels $x$ and $y$ is defined as product of two similarity measures of the normalized spectral reflectance and the spatial location. Using the Gaussian kernel function to represent the similarity measure, we describe

$$
w(x, y)=\exp \left(-\frac{\|\mathbf{F}(x)-\mathbf{F}(y)\|_{2}^{2}}{\sigma_{\mathrm{S}}^{2}}\right) * \exp \left(-\frac{\|\mathbf{Z}(x)-\mathbf{Z}(y)\|_{2}^{2}}{\sigma_{\mathrm{P}}^{2}}\right)
$$

where $\mathbf{F}(x) \in[0,1]^{N}$ is the $N$-dimensional feature vector at pixel $x$, which corresponds to the invariant representation $S^{\prime}(\lambda)$. The function $\mathbf{Z}(x)$ represents the spatial location that is effectively used to connect different regions to a similar segment, and ranges from 0 to the tested image size. The vector norm operator $\|\cdot\|_{2}$ is the Euclidean distance. The standard deviation $\sigma$ represents the sensitivity of the Gaussian distribution. The spectral sensitivity $\sigma_{\mathrm{S}}$ depends on the materials appearance while the spatial sensitivity $\sigma_{\mathrm{P}}$ depends on the size of tested image. The value of the $\sigma$ parameter is very important. The parameter $\sigma$ determines the distance of reachable neighbor pixels in one single step. A small $\sigma$ captures better the similarities of the pixels. However if $\sigma$ is too small, the pixels become disconnected. Thus, we need to optimize the spectral and the spatial sensitivity parameters $\sigma_{\mathrm{S}}$ and $\sigma_{\mathrm{P}}$.

Nyström approximation Exact normalized cut classification requires calculations of eigenvalues and eigenvectors of the huge similarity matrix $\mathbf{W}$. The Nyström method is a technique for finding a numerical approximation to the eigen decomposition, and it was widely applied to areas involving large dense matrices. Here the Nyström method estimates the eigenvalues and eigenvectors from smaller matrices. Let $L$ be the number of pixels. We empirically select number of sample pixels $m$, less than $1 \%$ of all data and more than number of segments, enough for choosing all objects in the tested image. Let $\mathbf{A} \in \mathbb{R}^{m \times{ }^{m}}$ represents the similarities amongst the sample pixels and $\mathbf{B} \in \mathbb{R}^{(L-m) \times m}$ represents the similarities between the $m$ sample pixels and the (L$m)$ remaining pixels. Two matrices $\mathbf{A}_{\mathrm{S}}$ and $\mathbf{B}_{\mathrm{S}}$ are constructed using $\|\mathbf{F}(x)-\mathbf{F}(y)\|_{2}$ from the invariant representation $S$ 'and two other matrices $\mathbf{A}_{\mathrm{P}}$ and $\mathbf{B}_{\mathrm{P}}$ are constructed using $\|\mathbf{Z}(x)-\mathbf{Z}(y)\|_{2}$ from the spatial location. Then, from the minimum average of the spectral matrices $\mathbf{A}_{\mathrm{S}}$ and $\mathbf{B}_{\mathrm{S}}$ and the spatial matrices $\mathbf{A}_{\mathrm{P}}$ and $\mathbf{B}_{\mathrm{P}}$, we estimate the spectral and the spatial sensitivity parameters as $\sigma_{\mathrm{S}}=\min \left\{\operatorname{avg}\left(\mathbf{A}_{\mathrm{S}}\right), \operatorname{avg}\left(\mathbf{B}_{\mathrm{S}}\right)\right\}$ and $\sigma_{\mathrm{P}}=\min \{\operatorname{avg}$ $\left.\left(\mathbf{A}_{\mathrm{P}}\right), \operatorname{avg}\left(\mathbf{B}_{\mathrm{P}}\right)\right\}$.

By using Equation 13, the two matrices of $\mathbf{A}$ and $\mathbf{B}$ are constructed from the estimated sensitivity parameters, and the spectral and the spatial matrices as $\mathbf{A}=\exp \left(-\mathbf{A}_{\mathrm{S}}^{2} / \sigma_{\mathrm{S}}^{2}\right) * \exp \left(-\mathbf{A}_{\mathrm{P}}^{2} / \sigma_{\mathrm{P}}^{2}\right) \quad$ and $\mathbf{B}=\exp \left(-\mathbf{B}_{\mathrm{S}}^{2} / \sigma_{\mathrm{S}}^{2}\right) * \exp \left(-\mathbf{B}_{\mathrm{P}}^{2} / \sigma_{\mathrm{P}}^{2}\right)$. Then the approximated eigenvectors matrix $\mathbf{V}$ is defined as

$$
\mathbf{V}=\left[\begin{array}{c}
\mathbf{A} \\
\mathbf{B}^{\mathrm{T}}
\end{array}\right] \mathbf{A}^{-1 / 2} \mathbf{U}_{\mathrm{Q}} \boldsymbol{\Lambda}_{\mathrm{Q}}^{-1 / 2},
$$

where $\mathbf{Q}=\mathbf{A}+\mathbf{A}^{-1 / 2} \mathbf{B B}^{\mathrm{T}} \mathbf{A}^{-1 / 2}$ and it is diagonalized as $\mathbf{Q}=\mathbf{U}_{\mathrm{Q}} \boldsymbol{\Lambda}_{\mathrm{Q}} \mathbf{U}^{\mathrm{T}}{ }_{\mathrm{Q}}$. We select the largest $k$ eigenvectors from matrix $\mathbf{V}$ according to the required number of $k$ materials.

K-means to cluster the leading Eigenvectors We use the $k$ eigenvectors stacked in columns simultaneously to get the final clusters for $k$ objects. From the matrix $\mathbf{V} \in$ $R^{L \times k}$ form matrix $\overline{\mathbf{V}}$ by renormalizing each of $\mathbf{V}$ 's rows to have unit length [34] as

$$
\overline{\mathbf{V}}_{i j}=\frac{\mathbf{V}_{i j}}{\sqrt{\sum_{j} \mathbf{V}_{i j}^{2}}}, \quad i=1, \ldots, L, j=1, \ldots, k .
$$


where $L$ is the number of pixels. By treating each row of the leading normalized eigenvectors $\bar{V}$ as a point in $R^{k}$, we cluster them into $k$ different objects via K-means that attempts to minimize the distortion. Finally, we assign the pixel $S_{\text {I }}$ of the normalized spectral reflectance to cluster $j$ if and only if row $i$ of the matrix $\overline{\mathbf{V}}$ was assigned to cluster $j$. Thus, the input normalized spectral reflectance data $S^{\prime}(\lambda)$ of spectral images is assigned to different segments to get the final segmentation.

\section{Experimental results}

We have examined the performance of the segmentation algorithm based on the proposed invariant representation for spectral images. First we used the same spectral reflectance image as in Figure 2. Figure 4a is the original image of a copper object and two dielectric objects. Figure $4 \mathrm{~b}$ depicts the ground truth of material classification by segmenting manually the image. Figure $4 \mathrm{c}$ shows the segmentation result using the original spectral reflectance data. The result contains many shadows and highlights effects. Figure $4 \mathrm{~d}$ shows the segmentation result using the proposed invariant representation. The image is clearly segmented into different material regions and background. Thus, the segmentation based on the proposed invariant representation is independent of the illumination effects of highlight and shadow, and the geometry of object shape.

In order to confirm the effectiveness of the invariant representation, two alternative methods were also examined. Although we tried several different parameter settings in each method, only the best results are shown for the comparison. The K-means classification method [35] was used to segment the spectral image in Figure 4a by clustering the original spectral reflectance data in the spectral space using random seeds, wherein the number of clusters was set to 4 . The result is shown in Figure 5a. The PCA + Ncut method [18] was used to execute the normalized cut in a low-dimensional space obtained with the principal components analysis. The segmentation result of clustering the original spectral reflectance data is shown in Figure $5 \mathrm{~b}$. We note that the



(a)



(c)

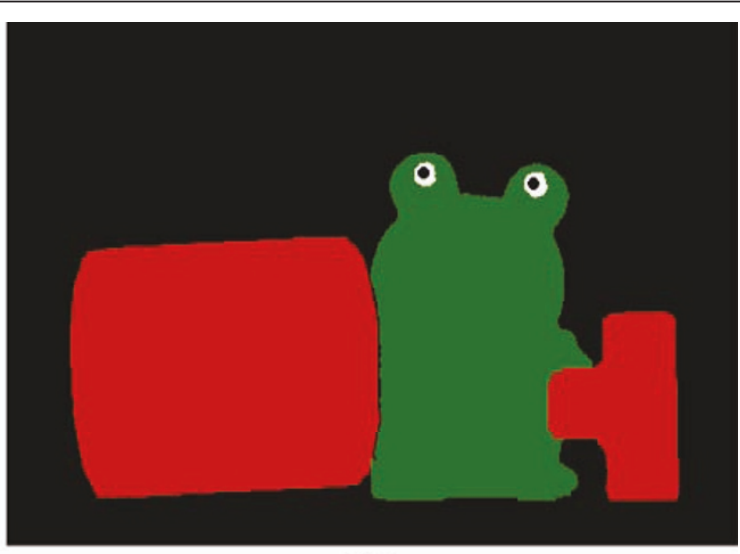

(b)

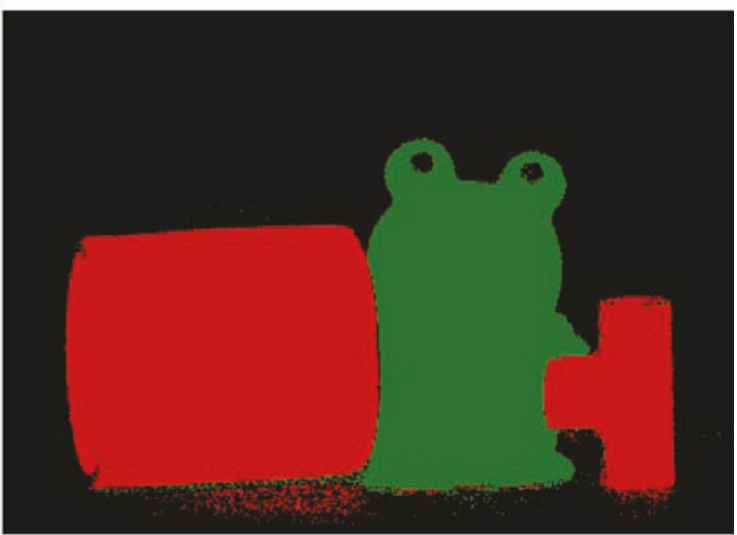

(d)

Figure 4 Evaluation of image segmentation for natural objects. (a) original image; (b) ground truth manually segmented; (c) segmentation result based on the original spectral reflectance data; (d) segmentation result based on the proposed invariant representation. 


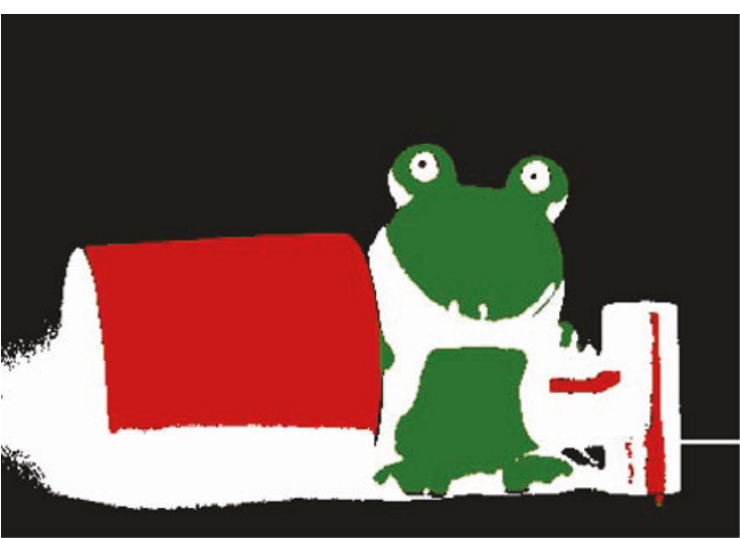

(a)



(c)



(b)



(d)

Figure 5 Segmentation results by using two alternative segmentation methods. (a) K-means [35] of the original spectral reflectance data; (b) PCA + Ncut [18] of the original spectral reflectance data; (c) K-means based on the proposed invariant representation; (d) PCA + Ncut based on the proposed invariant representation.

both segmentations are strongly affected by different illumination events on the objects surfaces. On the other hand, Figure $5 \mathrm{c}$, d shows the segmentation results by the K-means and the PCA + Ncut methods, respectively, based on the proposed invariant representation. The image is well segmented into different material regions and background, compared with the results in Figure $5 \mathrm{a}, \mathrm{b}$. However, the effect of inter-reflection appears in the segmented images.

Next, we have applied the proposed method to material classification of a printed circuit board with various tiny elements. A bare circuit board surface layer is composed of various elements, which are a mixture of different materials including dielectrics (i.e., photo-resist, silkscreen print, and substrate) and metals (i.e., copper), as shown in Figure 6. We used a part of a real circuit board. Figure 6a shows the color image of the original spectral reflectance data. The board is illuminated by one light source from the left direction. The size of the captured spectral image by our imaging system is $433 \times$ $363 \times 31$

Figure $6 \mathrm{~b}$ depicts the ground truth by manually segmenting the image. The ground truth image consists of four segments such as white-silk-screen print, yellowmetal, green-resist-coated metal, and black-background (substrate). Figure $6 \mathrm{c}$ shows the material classification result based on the original spectral reflectance data with the illumination effects. We find that miss classification occurs around metal and resist-coated metal lines at the left side of the segmented image. This can be caused by specular reflection of these materials surfaces. Figure $6 \mathrm{~d}$ shows the classification result on the proposed invariant representation without the illumination effects. The original scene is well segmented into four material regions.

The accuracy of the segmentation results between the segmented image and the ground truth image is 




(a)

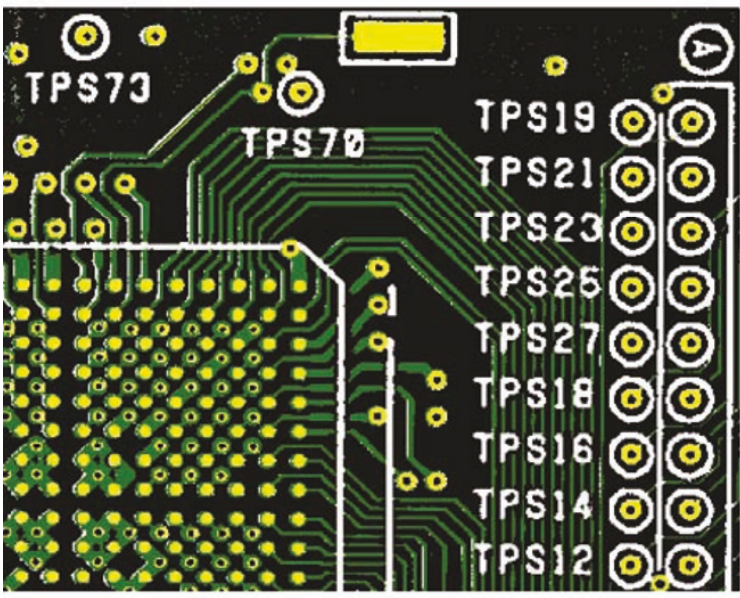

(c)



(b)

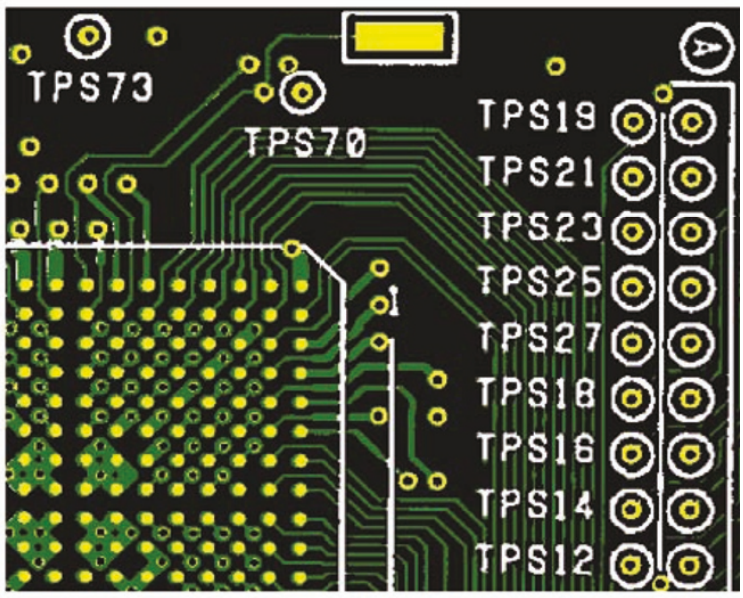

(d)

Figure 6 Material classification of a bare printed-circuit board. (a) color image of the original spectral reflectances; (b) ground truth image; (c) classification result based on the original spectral reflectance data; (d) classification result based on the proposed invariant representation.

numerically demonstrated by two different measures. First, we used the similarity measure with the window size $16 \times 16$ for labeled images shown in [36]. Second, a pixel by pixel comparison is used to calculate the segmentation quality numerically for the whole regions using

$$
\text { Quality rate }=\frac{\text { Correct classified pixels }}{\text { Total number of pixels }}
$$

Tables 1 and 2 list the numerical accuracy of segmentation results for the natural image and the circuit board image. The accuracy using different window size for the

Table 1 Accuracy of segmentation results for the natural scene.

\begin{tabular}{cc}
\hline Method and representation & Similarity measure (\%) \\
\hline Segmentation result based on the original spectral reflectance data & 95.76 \\
\hline Segmentation result based on the proposed invariant representation & 98.03 \\
\hline K-means using the original spectral reflectance data & 91.86 \\
\hline K-means using the proposed invariant representation & 93.85 \\
\hline PCA + Ncut using the original spectral reflectance data & 92.11 \\
\hline PCA + Ncut using the proposed invariant representation & 90.80 \\
\hline 9
\end{tabular}


Table 2 Accuracy of segmentation results for the printed circuit board.

\begin{tabular}{cll}
\hline Method and representation & $\begin{array}{l}\text { Similarity } \\
\text { measure (\%) }\end{array}$ & $\begin{array}{l}\text { Quality } \\
\text { rate (\%) }\end{array}$ \\
\hline $\begin{array}{c}\text { Segmentation result based on the } \\
\text { original spectral reflectance data }\end{array}$ & 86.02 & 91.51 \\
\hline $\begin{array}{c}\text { Segmentation result based on the } \\
\text { proposed invariant representation }\end{array}$ & 98.77 & 99.18 \\
\hline
\end{tabular}

similarity measure and the quality rate show that the segmentations based on the proposed invariant representation achieve high accuracy for both natural scene and circuit board scene, compared with the segmentation results using the original spectral reflectance data.

\section{Conclusions}

The present article has proposed an invariant representation for spectral reflectance images which can be applied to dielectric and metal objects. The invariant representation was derived from the standard dichromatic reflection model for dielectric and the extended dichromatic reflection model for metal. We have shown that the normalized surface-spectral reflectance by the minimum reflectance is invariant to highlights, shading, surface geometry, and illumination intensity. As an application of the proposed invariant representation, a segmentation algorithm based on the proposed representation was presented for effectively segmenting spectral images of natural scenes and bare circuit boards. Experimental results using real-world objects including metals and dielectrics showed that the proposed representation is invariant to highlight, shadow, and object surface geometry, and effective for image segmentation. We also explore as a future work the application of the proposed invariant representation in other fields and the effect of inter-reflection.

\section{List of Abbreviations}

HDR: high-dynamic range; LCT: liquid crystal tunable; Ncut: normalized cut; PCA: principal components analysis.

\section{Author details}

${ }^{1}$ Graduate School of Advanced Integration Science, Chiba University, 1-33, Yayoi-cho, Inage-ku, Chiba, 263-8522, Japan ${ }^{2}$ Computers and Systems Engineering Department, Faculty of Engineering, Mansoura University, Mansoura, Egypt

\section{Competing interests}

The authors declare that they have no competing interests.

Received: 26 July 2010 Accepted: 20 June 2011 Published: 20 June 2011

\section{References}

1. Trémeau A, Tominaga S, Plataniotis $\mathrm{KN}$ : Color in image and video processing: most recent trends and future research directions. EURASIP J Image Video Process 2008, 26
2. Burghouts GJ, Geusebroek J-M: Performance evaluation of local colour invariants. Comput Vision Image Understand 2009, 113(1):48-62.

3. Geusebroek J-M, Boomgard RV, Smeulders AWM, Geerts H: Color invariance. IEEE Trans Pattern Anal Machine Intell 2001, 23(12):1338-1350.

4. van de Weijer J, Gevers T, Geusebroek J-M: Edge and corner detection by photometric quasi-invariants. IEEE Trans Pattern Anal Mach Intell 2005, 27(4):625-630

5. van de Weijer J, Gevers T, Smeulders AWM: Robust photometric invariant features from the color tensor. IEEE Trans Image Process 2006, 15(1):118-127

6. Abdel-Hakim AE, Farag AA: CSIFT: A SIFT descriptor with color invariant characteristics. Proceedings of the IEEE Computer Society Conference on Computer Vision and Pattern Recognition (CVPR'06) 2006, 1978-1983.

7. Stokman HMG, Gevers T: Detection and classification of hyper-spectral edge. Proceedings of 10th British Machine Vision Conference (BMVC'99) 1999, 643-651.

8. Montoliu R, Pla F, Klaren AK: Illumination Intensity, object geometry and highlights invariance in multispectral imaging. In Proceedings of 2 nd Iberian Conference on Pattern Recognition and Image Analysis (IbPRIA2005). Volume 3523. LNCS; 2005:36-43.

9. Shafer SA: Using color to separate reflection components. Color Res Appl 1985, 10(4):210-218.

10. Lee HC, Breneman EJ, Schulte C: Modeling light reflection for computer color vision. IEEE Trans Pattern Anal Mach Intell 1990, 12(4):402-409.

11. Tominaga S, Wandell B: Standard reflectance model and illuminant estimation. J Opt Soc Am A 1989, 6(4):576-584

12. Tominaga S: Surface identification using the dichromatic reflection model. IEEE Trans Pattern Anal Mach Intell 1991, 13(7):658-670.

13. Tominaga S: Dichromatic reflection models for a variety of materials. Color Res Appl 1994, 19(4):277-285.

14. Tominaga S: Dichromatic reflection models for rendering object surfaces. J Imag Sci Technol 1996, 40(6):549-555.

15. Paclík P, Duin RPW, van Kempen GMP, Kohlus R: Segmentation of multispectral images using the combined classifier approach. Image Vision Comput 2003, 21(6):473-482.

16. Martínez-Usó A, Pla F, García-Sevilla P: Multispectral image segmentation by energy minimization for fruit quality estimation. In Proceedings of 2 nd Iberian Conference on Pattern Recognition and Image Analysis (IbPRIA2005) Volume 3523. LNCS; 2005:689-696

17. Mohammad-Djafari A, Bali N: A Mohammadpour, Hierarchical Markovian models for hyperspectral image segmentation. Proceedings of International Workshop on Intelligent Computing in Pattern Analysis/Systems (IWICPAS) 2006, 416-424.

18. Ibrahim A, Tominaga $\mathrm{S}$, Horiuchi $\mathrm{T}$ : Unsupervised material classification of printed circuit boards using dimension-reduced spectral information. Proceedings of IAPR Conference on Machine Vision Applications (MVA2009) 2009, 435-438

19. Ibrahim A, Tominaga $S$, Horiuchi T: Spectral imaging method for material classification and inspection of printed circuit boards. Opt Eng 2010, 49(5):057201-10

20. Moganti F, Ercal F, Dagli CH, Tsunekawa S: Automatic PCB inspection algorithms: a survey. Comput Vision Image Understand 1996, 63(2):287-313.

21. Chang PC, Chen LY, Fan CY: A case-based evolutionary model for defect classification of printed circuit board images. J Intell Manuf 2008, 19(2):203-214

22. Tsai DM, Yang RH: An eigenvalue-based similarity measure and its application in defect detection. Image Vision Comput 2005, 23(12):1094-1101.

23. Huang SY, Mao CW, Cheng KS: Contour-based window extraction algorithm for bare printed circuit board inspection. IEICE Trans 2005, 88D(12):2802-2810.

24. Morimoto T, Ikeuchi $\mathrm{K}$ : Multispectral imaging for material analysis in an outdoor environment using normalized cuts. Proceedings of IEEE Color and Reflectance in Imaging and Computer Vision Workshop (CRICV2009), in conjunction with ICCV'09 2009, 1909-1916.

25. Ibrahim A, Tominaga S, Horiuchi T: Material classification for printed circuit boards by spectral imaging system. In Proceedings of IAPR Computational Color Imaging Workshop (CCI'09). Volume 5646. LNCS. 2009:216-225.

26. Tominaga S: Spectral imaging by a multi-channel camera. J Electron Imag 1999, 8(4):332-341 
27. Hardeberg JY, Schmitt F, Brettel H: Multispectral color image capture using a liquid crystal tunable filter. Opt Eng 2002, 41(10):2532-2548.

28. Shi J, Malik J: Normalized cuts and image segmentation. IEEE Trans Pattern Anal Mach Intell 2000, 22(8):888-905.

29. Fowlkes C, Belongie S, Chung FRK, Malik J: Spectral grouping using the Nyström method. IEEE Trans Pattern Anal Mach Intell 2004, 26(2):214-225.

30. Tominaga S: Multichannel vision system for estimating surface and illumination functions. J Opt Soc Am A 1996, 13(11):2163-2173.

31. Parkkinen JPS, Hallikaine J, Jaaskelainen T: Characteristic spectra of Munsell colors. J Opt Soc Am A 1989, 6(2):318-322.

32. Tominaga S, Fukuda T, Kimachi A: A high-resolution imaging system for omnidirectional illuminant estimation. J Imag Sci Technol 2008, 52(4):040907-9.

33. Tominaga S, Matsuura A, Horiuchi T: Spectral analysis of omnidirectional illumination in a natural scene. J Imag Sci Technol 2010, 54(4):040502-9.

34. Ng AY, Jordan Ml, Weiss Y: On spectral clustering: analysis and an algorithm. Proceedings of Advances in Neural Information Processing Systems (NIPS) 2001, 849-856.

35. Duda RO, Hart PE, Stork DG: Pattern Classification. 2 edition. John Wiley \& Sons, New York; 2001.

36. Horiuchi T: Similarity measure of labelled images. Proceedings of IAPR 17th International Conference on Pattern Recognition (ICPR2004) 2004, 3:602-605.

doi:10.1186/1687-5281-2011-2

Cite this article as: Ibrahim et al.: Invariant representation for spectral reflectance images and its application. EURASIP Journal on Image and Video Processing 2011 2011:2.

\section{Submit your manuscript to a SpringerOpen ${ }^{\circ}$ journal and benefit from:}

- Convenient online submission

- Rigorous peer review

- Immediate publication on acceptance

- Open access: articles freely available online

- High visibility within the field

- Retaining the copyright to your article

Submit your next manuscript at $\gg$ springeropen.com 